\title{
Unveiling multiple AGN activity in galaxy mergers
}

\author{
A. De Rosa ${ }^{1, \star}$ S. Bianchi ${ }^{2}$, T. Bogdanović ${ }^{3}$, R. Decarli ${ }^{4}$, J. Heidt $^{5}$ R. Herrero-Illana $^{6,7}$, B. Husemann ${ }^{8,9}$, \\ S. Komossa ${ }^{10}$, E. Kun ${ }^{11}$, N. Loiseau ${ }^{12}$, M. Guainazzi ${ }^{13}$ Z. Paragi $^{14}$, M. Perez-Torres ${ }^{6,15}$, E. Piconcelli ${ }^{16}$, \\ K. Schawinski ${ }^{17}$, C. Vignali $^{18}$ \\ 1 INAF/Istituto di Astrofisica e Planetologie Spaziali. Via Fosso del Cavaliere - 00133 Roma - Italy \\ 2 Dipartimento di Matematica e Fisica, Università degli Studi Roma Tre, via della Vasca Navale 84, 00146 Roma, Italy \\ 3 Center for Relativistic Astrophysics, Georgia Institute of Technology, Atlanta, GA 30332, USA \\ 4 Max-Planck Institut fuer Astronomie, Germany \\ 5 ZAH Landessternwarte Knigstuhl. D-69117 Heidelberg. Germany \\ 6 Instituto de Astrofísica de Andalucía (IAA-CSIC), 18008 Granada, Spain \\ 7 European Southern Observatory (ESO), Alonso de Cordova 3107, Vitacura, Casilla 19001, Santiago de Chile, Chile \\ 8 MPIA, Konigstuhl 17 D-69117 Heidelberg, Germany \\ 9 European Southern Observatory Headquaters, Karl-Schwarzschild-Str. 2, 85748 Garching, Germany \\ 10 QianNan Normal University for Nationalities, Longshan Street, Duyun City of Guizhou Province, China \\ 11 Department of Experimental Physics, University of Szeged, Dóm tér 9, H-6720 Szeged, Hungary \\ 12 ESAC/XMM-Newton Science Operations Centre, Spain \\ 13 ESA - European Space Agency. ESTEC, Keplerlaan 1 2201AZ Noordwijk, The Netherlands \\ 14 Joint Institute for VLBI ERIC, Postbus 2, NL-7900 AA Dwingeloo, The Netherlands \\ 15 Visiting Scientist at the Depto. de Física Teórica, Facultad de Ciencias, Univ. de Zaragoza, Spain \\ 16 Osservatorio Astronomico di Roma (INAF), via Frascati 33, 00040 Monte Porzio Catone ( Roma), Italy \\ 17 Institute for Astronomy, Wolfgang-Pauli-Str. 27, 8093 Zrich, Switzerland \\ 18 Dipartimento di Fisica e Astronomia, Università degli studi di Bologna, Viale Berti Pichat 6/2, 40127, Bologna, Italy
}

Received 4 October 2016, accepted 4 October 2016

Key words galaxies: active-galaxies: Seyfert-galaxies: interactions-X-rays: galaxies

In this paper we present an overview of the MAGNA (Multiple AGN Activity) project aiming at a comprehensive study of multiple supemassive black hole systems. With the main goal to characterize the sources in merging systems at different stages of evolution, we selected a sample of objects optically classified as multiple systems on the basis of emission line diagnostics and started a massive multiband observational campaign. Here we report on the discovery of the exceptionally high AGN density compact group SDSS J0959+1259. A multiband study suggests that strong interactions are taking place among its galaxies through tidal forces, therefore this system represents a case study for physical mechanisms that trigger nuclear activity and star formation. We also present a preliminary analysis of the multiple AGN system SDSS J1038+3921.

(C) 2016 WILEY-VCH Verlag GmbH \& Co. KGaA, Weinheim

\section{Introduction}

Hierarchical merger models of galaxy formation predict that binary AGN should be common in galaxies (Haehnelt et al. 2002; Volonteri et al. 2003). Understanding the types of galaxies and specific merger stages where AGN pairs occur provides important clues about the peak of the black hole growth during the merging process (Begelman et al.1980; Escala et al. 2004). First compact AGN pairs in advanced mergers and interacting galaxies have been identified in Xrays (e.g., Komossa et al. 2003, Bianchi et al. 2008, Piconcelli et al. 2010, Guainazzi et al. 2005) and in the radio band (Rodriguez et al. 2006). Given the small number of known systems, there is a need of increasing the sample size significantly, in order to cover a wide range of spa-

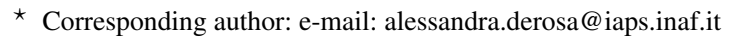
www. issibern.ch/teams/agnactivity/Home.html
}

tial separations and stages of galaxy mergers. Therefore, the search for AGN pairs has recently received great attention (see Bogdanovic 2015 and Komossa \& Zensus 2016 for recent reviews), and different methods have been proposed to identify good candidates. With the availability of massive spectroscopic surveys like the Sloan Digital Sky Survey (SDSS), candidate AGN pairs have been identified from optical double-peaked emitters. In these systems, the narrow line emission is double-peaked, and one possible interpretation is that they consist of a pair of AGN. Multi-wavelength follow-up campaigns have shown that only a small fraction of these systems actually harbour AGN pairs (e.g., Fu et al. 2011, Fu et al. 2012, Smith et al. 2012, Comerford et al. 2012), while the majority of the double-peakers is produced by other mechanisms like two-sided outflows, or jet-cloud interactions. Follow-up observations of candidate pairs in the infrared, optical, radio and X-rays are therefore essential in finding the true pairs. 
The key goal of our MAGNA (Multiple AGN Activity) project is to determine the multiband properties (radio, IR, optical and X-rays) of a homogeneously selected sample of AGN systems in different stages of merging. As a first step, we identified the systems from the sample of AGN pairs optically selected from the SDSS-DR7 by Liu et al. (2011). We selected the optically classified Seyfert-Seyfert pairs using the $[\mathrm{OIII}] / \mathrm{H} \beta$ line-ratio diagnostic (Baldwin et al. 1981). Finally, we chose the maximum projected separation of a pair of sources to be $<60 \mathrm{kpc}$ to remove from the sample many non-interacting AGN pairs (Satyapal et al. 2014). This separation threshold allows us to keep a significant number of wide pairs. The final "master sample" consists of 16 systems, all of them harbouring at least two AGN (Sy-Sy type, following the optical classification) with redshift between 0.03-0.17. We started intensive observational campaigns (VLA, EVN, MUSE, XMM) on the master sample.

In this paper we report on the study of multiple SMBH systems investigated in our MAGNA programme. Data analysis is presented in Sect. 2. In Sect. 3 we report on the discovery of the exceptionally high AGN density compact group SDSS J0959+1259 while a preliminary analysis of the multiband data of the system SDSS J1038+3921 is presented in Sect. 4. Our conclusions and future prospects are described in Sect. 5 We assume $\mathrm{H}_{0}=70 \mathrm{~km} \mathrm{~s}^{-1} \mathrm{Mpc}^{-1}$, $\Omega_{\Lambda}=0.7, \Omega_{M}=0.3$, and AB magnitudes.

\section{MAGNA: Observations and data analysis}

\subsection{X-rays}

The sources in the systems observed with XMM-Newton were detected using the EPIC source finding threads edetect chain, on 5 images in the $0.3-0.5 \mathrm{keV}, 0.5-1$ $\mathrm{keV}, 1-2 \mathrm{keV}, 2-4.5 \mathrm{keV}, 4.5-12 \mathrm{keV}$ energy bands with a detection threshold of $3 \sigma$. All spectra were extracted from circular regions (with radius ranging from 15" to 30 " depending on the source counts) which include more than $80 \%$ of the source counts at $1.5 \mathrm{keV}$ in the EPIC cameras. The background spectra were extracted in the same CCD chip from circular regions free from contaminating objects and of the same size as the regions containing the source. All events were screened and filtered for the flaring events. Each spectrum (and associated background) was rebinned in order to have at least 25 counts for each backgroundsubtracted spectral channel and not to oversample the intrinsic energy resolution by a factor larger than 3. Spectral fits for pn and (co-added) MOS cameras were performed in the $0.3-10 \mathrm{keV}$ energy band.

\subsection{Optical}

In order to identify the multiple sources in the systems we used the most common optical emission-line diagnostic diagrams (BPT, Baldwin et al. 1981). We retrieved the SDSSIII DR12 spectra for all sources in our systems (see Tab.
1 from the survey webpage 1 . The emission line flux of all the primary diagnostic lines $(\mathrm{H} \beta,[\mathrm{OIII}] \lambda 5007,[\mathrm{OI}] \lambda 6300$, $\mathrm{H} \alpha$, [NII] $\lambda 6583$ and [SII] $\lambda \lambda 6713,6732)$ were measured on top of the stellar continuum using the package PYPARADISE. PYPARADISE models the stellar continuum as a superposition of template stellar population spectra from the CB07 library Bruzual \& Charlot 2003 after normalizing both the SDSS and the template spectra with a running mean over 100pix, interpolating regions with strong emission lines. A simple Gaussian kernel is used to match the template spectra to the line-of-sight velocity distribution. The line fluxes are then inferred by fitting the Gaussian line profiles coupled in redshift and intrinsic rest-frame velocity dispersion. Errors are obtained using a bootstrap approach where 100 realizations of the spectrum were generated based on the pixel errors with just $80 \%$ of the template spectra and modelled again with the same approach (at fixed stellar kinematics).

In order to better characterize the sources in the crowded field of SDSS J0959+1259 we observed the region using the multiband camera BUSCA on the $2.2 \mathrm{~m}$ telescope in Calar Alto observatory. These observations span the whole optical window from $\mathrm{U}$ to I over a $12^{\prime} \times 12^{\prime}$ field of view. The throughput curves of the dichroic mirrors, convolved with the detector efficiency, roughly correspond to those of the SDSS u, r, i+z, and Johnson B filters. Five 12 minlong frames were collected in each band, dithered by a few arcsec in order to clean our final images from cosmic rays and bad pixels. Full details on data analysis of the system SDSS J0959+1259 are reported in De Rosa et al. 2015.

\subsection{Radio}

We used radio $1.4 \mathrm{GHz}$ data from two public data sets: (1) the NRAO VLA Sky Survey (NVSS; Condon et al. 1998), which have an angular resolution of $\sim 45$ arcsec and a completeness limit of $2.5 \mathrm{mJy}$, and (2) the Faint Images of the Radio Sky at Twenty-Centimeters Survey (FIRST; Becker et al. 1994) with a resolution of $\sim 5$ arcsec and a typical rms of $0.15 \mathrm{mJy}$.

\section{The Compact Group SDSS J0959+1259}

The system associated with the SDSS J0959+1259 at redshift $\mathrm{z}=0.035$ was recognized as the only quintuplet detected in a sample of 1286 multiple AGN/LINER systems (Liu et al. 2011). The galaxies in this field that constitute a compact group (CG) have projected separations of $\lesssim 100 \mathrm{~h}^{-1}$ $\mathrm{kpc}$ and the line-of-sight velocity differences of $\lesssim 500 \mathrm{~km}$ $\mathrm{s}^{-1}$ (Hickson 1997). Within these criteria we find 7 spectroscopically identified sources (from I-src1 to I-src7 in Tab. 11) while another source in this region (I-src8 NGC 3080) is close in redshift $(\mathrm{z}=0.035)$ but at larger projected separation, and it is included in our study. Detailed study on

\footnotetext{
1 http://skyserver.sdss3.org/dr12
} 

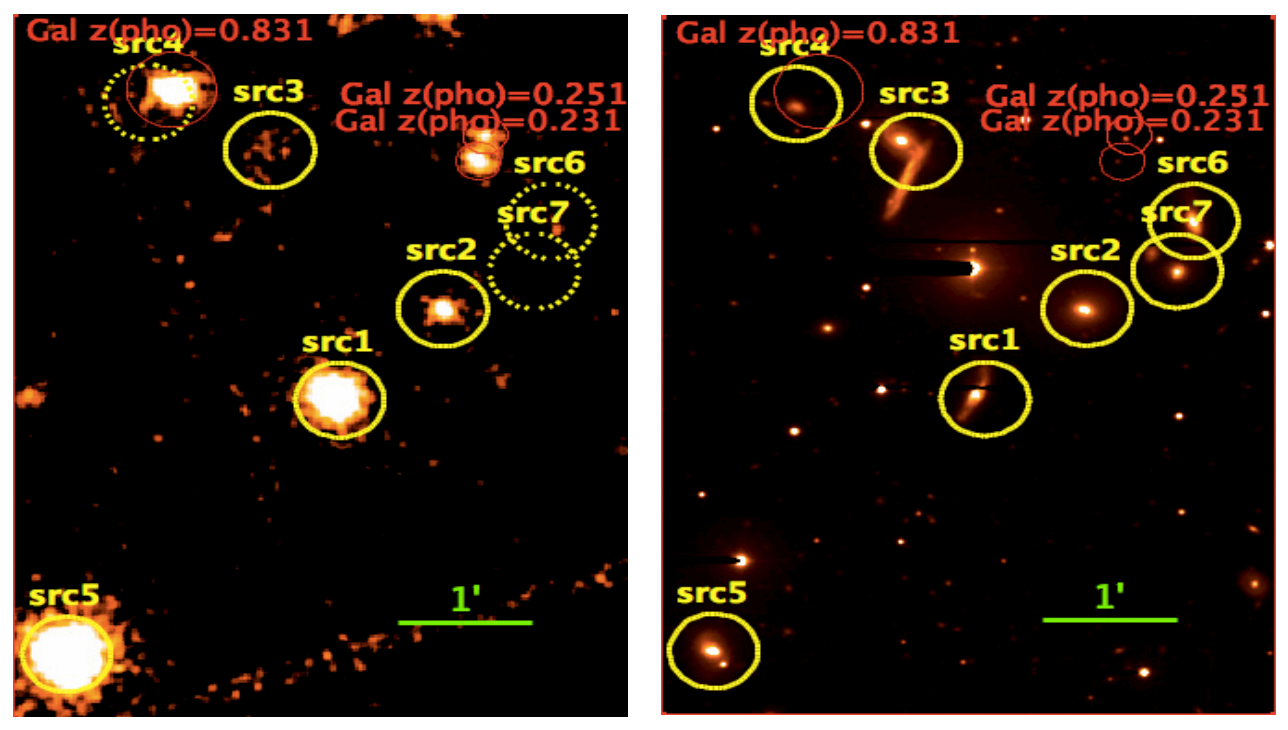

Fig. 1 Left: XMM-EPIC smoothed mosaic image of the CG region (pn, MOS1 and MOS2 co-added). North is up and East to the left. I-src8 is off the plot, 10 arcmin to the East from src1. Yellow circles show the group sources while undetected sources are shown with a dashed line. Red circles are the sources in the field not included in our group lacking a spectroscopic redshift in the SDSS (our estimated photometric redshifts based on BUSCA images suggest that they are background objects). Right: The BUSCA R-band image of the same region on the left. Labels are the same.

SDSS J0959+1259 is presented in De Rosa et al. (2015). We have analysed the multiwavelength properties of the system using radio, optical and X-ray data. The mean redshift of the CG is $\langle z\rangle=0.0353$ and the mean velocity is $\langle\mathrm{v}\rangle=10593 \mathrm{~km} \mathrm{~s}^{-1}$. The center of this field has RA $09 \mathrm{~h}$

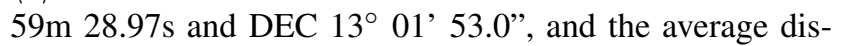
tance of sources from the geometric center of the group is $240 \mathrm{kpc}$. The CG is shown in Fig. 11, where the composite EPIC-pn and MOS12 (left panel) and the R-band BUSCA images (right panel) are presented. I-src8, which is located 10 arcmin to the East from src1, is not shown. Yellow circles indicate CG sources, the dashed yellow circles mark the three objects not detected in X-rays, while the red circles show the background sources in the field not related to the group. All three BPT diagrams lead to a consistent classification of objects into two Seyfert 2s, two LINERs and three Star Forming Galaxies (SFGs). The optical spectrum of I-src8 shows broad Balmer emission lines (FWHM $\sim 750$ $\mathrm{km} \mathrm{s}^{-1}$ ) and thus points to a type-1 Seyfert. The 0.3-10 $\mathrm{keV}$ spectra of the two Seyfert 2s (I-src1 and I-src5) are modelled with an absorbed power-law plus a thermal component with temperature of $\mathrm{kT} \sim 0.1-0.6 \mathrm{keV}$ (mekal in $\mathrm{Xspec}$ ) emerging below $2 \mathrm{keV}$. The cold absorption gas has column densities in the range $\mathrm{N}_{H} \approx 1-20 \times 10^{22} \mathrm{~cm}^{-2}$. The broadband spectrum the Seyfert 1 (I-src8) is well fitted with an absorption component with partial covering fraction $\mathrm{f}_{c}=0.41 \pm 0.07$. The range of luminosity of the Seyferts is $10^{42}-10^{43} \mathrm{erg} \mathrm{s}^{-1}$ and a narrow $\mathrm{Fe} \mathrm{K} \alpha$ emission line is also measured in their spectra, with an equivalent width of $\sim 100-130 \mathrm{eV}$. The two LINERs (I-src2 and I-src3) are modelled with an unabsorbed power-law continuum in the X-ray

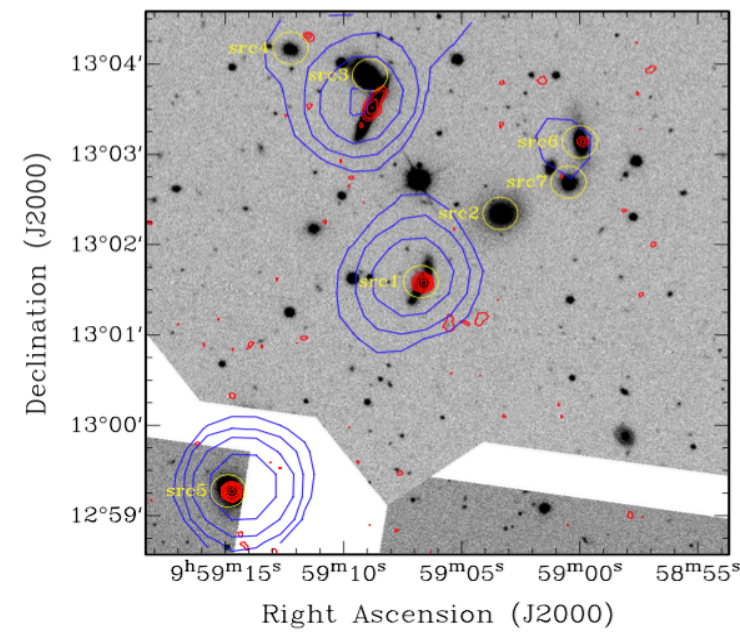

Fig. 2 SDSS J0959+1259. An overlay of the SDSS images of the compact group with the Very Large Array NVSS (blue contours) and FIRST (red contours) survey maps.

band and show no detectable Fe line. Their $2-10 \mathrm{keV}$ luminosity is nevertheless high $\left(\sim 10^{41} \mathrm{erg} \mathrm{s}^{-1}\right)$, above the mean value found in the systematic X-ray study of the largest sample of LINERs (González-Martínez et al. 2009). This is a strong indication that LINERs in this CG may be accretion driven; in this case, the fraction of AGN in the CG rises from 40 to $60 \%$ (from 3 to 5 out of 8 ). None of the SFGs (I-src4, I-src6, I-src7) are detected by XMM, placing a $3 \sigma$ upper limit on flux in $2-10 \mathrm{keV}$ of about $1.4 \times 10^{-14} \mathrm{erg} \mathrm{cm}^{-2} \mathrm{~s}^{-1}$. This corresponds to a luminosity of $\sim 4 \times 10^{40} \mathrm{erg} \mathrm{s}^{-1}$, assuming a photon index 1.7 and absorbing column density 
of $10^{22} \mathrm{~cm}^{-2}$. In order to complement the multiwavelength information, we analyzed radio archival data, an overlay of SDSS optical as well as VLA NVSS and FIRST survey images is shown in Fig. 2. The two Sy2 AGN (I-src 1 and I-src5) are both detected at the 10-20 mJy level, and the FIRST/NVSS ratios indicate these are quite compact on arcsec scales. The third well-established AGN (I-src 8) is indicating a resolved radio emission already on arcsec scales. The two LINERs are undetected on $\sim$ mJy levels, and only one SFG is detected in FIRST but not in NVSS. A comparison of radio-to-X-ray luminosities indicates that the two Sy2s are extremely radio-loud, and the Sy1 is radio-quiet, i.e. below $\log \mathrm{R}_{X}=-4.5$, with $\mathrm{R}_{X}=\mathrm{L}_{R} / \mathrm{L}_{X}$ (Terashima \& Wilson 2003).

The CG in SDSS J0959+1259 represents one of the best examples of exceptionally strong nuclear activity in CGs in the nearby Universe. The only other example of an AGN group rich like this is the well known HCG 16 (Ribeiro 1996 Turner et al. 2001), with an AGN fraction of $75 \%$ (three out of four galaxies). In addition, the clearly distorted shape of one member galaxy (I-src1) also hints on strong interactions within the group. The high AGN fraction in the $\mathrm{CG}$ and the signature of interactions make therefore SDSS J0959+1259 ideally suited to study nuclear activity and star formation triggered by tidal interactions in compact groups. We then started a massive observational campaign on this $\mathrm{CG}$, obtaining high resolution EVN + eMERLIN and VLT-MUSE observations. These combined observations will reveal further inside into the cause of the exceptionally strong activity of this CG and what it implies for the evolution of its members (see details in Sect. 5).

\section{The multiple SMBH system in SDSS J1038+3921}

The main characteristics of the system in SDSS J1038+3921 are reported in Tab. 1. From the optical spectrum, the objects in the system have been classified as a BL AGN (II-src1) and a NL AGN (II-src2). SDSS spectra for both sources are shown in Fig. 3, while the X-ray image of SDSS J1038+3921 is shown in Fig. 4. The bright source (II-src1) clearly dominates the emission in the whole band, however the presence of II-src2 is evident mainly in the soft band (0.3-2 keV). The X-ray spectrum of the BL AGN (IIsrc1) is shown in the left panel of Fig. 5 and is reproduced with a primary power-law emission with cold and possibly warm absorber and an intrinsic $\mathrm{L}(2-10 \mathrm{keV})=3 \times 10^{42}$ erg $\mathrm{s}^{-1}$. A Fe $\mathrm{K}$ line at $6.4 \mathrm{keV}$ with equivalent width of about $200 \mathrm{eV}$ is also detected. The X-ray spectrum of IIsrc2 is shown in the right panel of Fig. 5 and is well reproduced with a highly absorbed power-law and a soft component emerging below $2 \mathrm{keV}$ that is possibly due to photoionized emission (Guainazzi \& Bianchi 2007). The observed $2-10 \mathrm{keV}$ luminosity is about $10^{41} \mathrm{erg} \mathrm{s}^{-1}$. Due to the limited photon statistics in $2-10 \mathrm{keV}$ and the XMM energy band limited to $10 \mathrm{keV}$, we are not able to con- straint the absorption column density, then, in order to better characterize the nature of absorption in II-src2, we analysed archival multiband data and looked for absorption diagnostic. Fitting the optical spectrum of II-src2 we measure $\mathrm{L}([\mathrm{OIII}])$ corrected for extinction of $2 \times 10^{41} \mathrm{erg} \mathrm{s}^{-1}$, similar to the observed $\mathrm{L}(\mathrm{X})$. The evidence $(\mathrm{L}(\mathrm{X}) / \mathrm{L}([\mathrm{OIII}]) \lesssim 1)$ may be strongly suggestive of a deep absorption (see Cappi et al. 2006). Assuming a cold Compton-thick absorber (i.e. $\mathrm{N}_{H}=10^{24} \mathrm{~cm}^{-2}$ ) we obtain an intrinsic luminosity in (2$10 \mathrm{keV}$ ) of about $10^{42} \mathrm{erg} \mathrm{s}^{-1}$. The fit of the optical-tomid-IR spectral energy distribution (SED) of II-src2 with a SED-decomposition code (including stellar and AGN components; see Fritz et al. 2006; Feltre et al. 2012) provides a $12 \mu \mathrm{m}$ of $\approx 2.8 \times 10^{42} \mathrm{erg} \mathrm{s}^{-1}$ for the AGN component. This converts into a predicted, intrinsic $2-10 \mathrm{keV}$ luminosity of $\approx 2.5 \times 10^{42} \mathrm{erg} \mathrm{s}^{-1}$ assuming the Gandhi et al. (2009) relation, in a very good agreement with $\mathrm{L}(\mathrm{X})$ we have measured in the case of deep absorption, strengthening the hypothesis of a Compton thick source (see e.g. Lanzuisi et al. 2015).

From our multiband analysis we consider the more likely scenario of II-src 2 as a bona-fide CT AGN in which the $>10 \mathrm{keV}$ emission is not detected. However, we cannot ignore another (even more intriguing) possibility of a shutoff AGN, i.e. that we are observing in the optical waveband the past activity of a now quiet BH. It is worth noting that in all the X-ray reflection-dominated sources the only possible way to exclude this alternative hypothesis is either see the primary continuum piercing at hard X-rays (i.e. above 10 $\mathrm{keV}$ ), or detect an unveiling event due to absorption variability. The fact that the other AGN is unabsorbed is also intriguing, somehow different with respect to most of the AGN pairs observed in X-rays (Bianchi et al. 2008, Koss et al. 2011, Guainazzi et al. 2005).

\section{Future prospects in the next XMM and Chandra decade}

We have presented our MAGNA programme that aims at studying the multiband properties of a well selected sample of multiple AGN in different stages of merging. We have discovered the CG SDSS J0959+1259 characterized by exceptionally strong activity (five out eight galaxies are accretion powered). Further studies of this CG are still on-going. At a mean redshift of $z=0.0353$, the NVSS and FIRST radio sensitivities already allowed us to measure/constrain radio powers in the $10^{21-22} \mathrm{~W} \mathrm{~Hz}^{-1}$ regime. Our approved EVN observation will go more than an order of magnitude deeper in sensitivity allowing us to detect compact emission from the two LINERs (I-src2 and I-src3 in Tab. 1] Detection of these sources on 10-mas scales is expected because partially self-absorbed compact jets are ubiquitous in LINERs according to VLBI studies, confirming that at least half of these are accretion-powered (Nagar et al. 2005); in fact, the high X-ray luminosities of I-src2 and I-src3 are consistent with this prediction. The EVN and eMERLIN observations at $1.7 \mathrm{GHz}$ will yield spatial resolutions of 

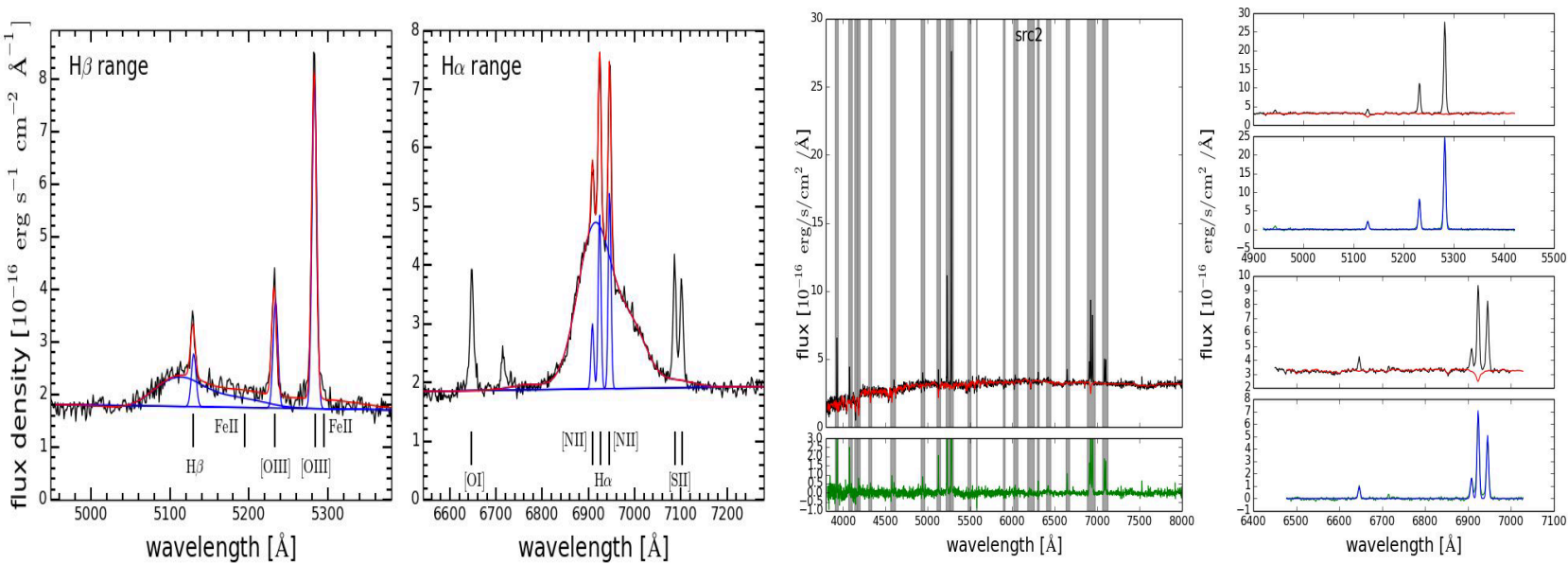

Fig. 3 SDSS J1038+3921. The SDSS spectra of the BL AGN (src1, left panels) and the NL AGN (src2, right panels). For the NL we first model the stellar continuum with a super-position of single-stellar population templates and a simple Gaussian kernel for the line-of-sight kinematics. Then we fit the lines with single Gaussians coupled in the kinematics. For the BL we subtract a quasi-linear continuum from the adjacent continuum of the broad Balmer lines. Afterwards we fit a high-order Gauss-Hermite polynom to the broad component and a double Gaussian for the narrow lines but coupling all lines with the same kinematics.

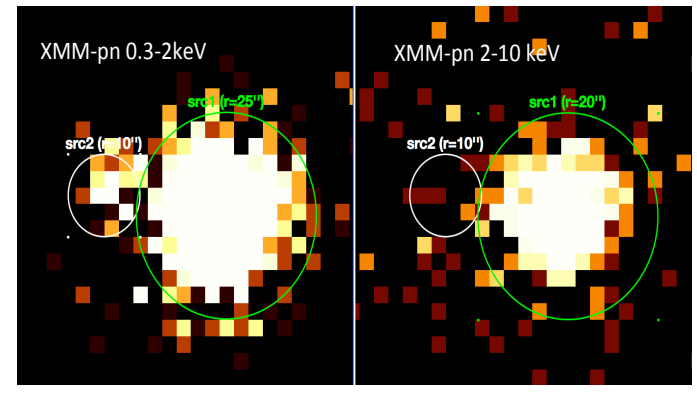

Fig. 4 SDSS J1038+3921. Left: XMM-pn map in 0.3-2 keV (left panel) and 2-10 keV (right panel).

about $3 \mathrm{pc}$ and $100 \mathrm{pc}$, respectively. Therefore, our observations will allow us to trace the pc-scale of the jet from $\sim 3-$ $100 \mathrm{pc}(\mathrm{eEVN})$, while eMERLIN will trace this emission above $100 \mathrm{pc}$ scales. Our combined EVN-eMERLIN imaging will permit us to obtain an unprecedented view of the synchrotron emission powering each individual AGN in this remarkable compact group from the pc- to the kpc-scale. We have also obtained integral-field spectroscopy data with MUSE on the VLT; these complement our high-resolution radio follow-up, as we will directly probe the impact of the jet on the kinematics of the ionized gas and compare the $\mathrm{H} \alpha$-based star formation rates locally (jet-induced star formation) and globally. The deep IFU spectroscopy will allow us to map all galaxies and beyond to understand the cause for the enhanced activity of the group. In particular we will measure kinematic disturbances in the stellar and ionized gas kinematics, the stripped gas and bridges in relation to nuclear activity and star formation rate.

It is worth noting that the high penetrative power of hard
$\mathrm{X}$-rays provides a unique and often ultimate tool in the hunt for multiple active nuclei in a galaxy, being less affected by contamination and absorption, and produced in large amounts only by AGN. The high point like luminosity, the spectral shape of the continuum and the presence of a strong Fe line emission in the $\mathrm{X}$-ray spectrum are clear signatures of the presence of an AGN with respect to a region of starburst emission. The clearest cases are the Chandra detected double nucleus in the luminous infrared galaxy NGC 6240 (Komossa et al. 2003) with a projected separation $\mathrm{d} \sim 1 \mathrm{kpc}$, Mrk 463 (d 3.8 kpc, Bianchi et al. 2008), Mrk 739 (d 3.4 kpc, Koss et al. 2011); and the XMM-Newton detection of IRAS 20210+1121 (d 11 kpc, Piconcelli et al. 2010), ESO509-IG066 (d 10.5 kpc , Guainazzi et al. 2005), and possibly AM1211-465 (d 98 kpc, Jiménez-Bailón, 2007). Some members of these systems show no (or very weak) explicit evidence of AGN in their optical/near-infrared spectra. Together with SDSS J1038+3921, the XMM-OTAC granted to our team in AO15 3 additional systems (4 sys- 

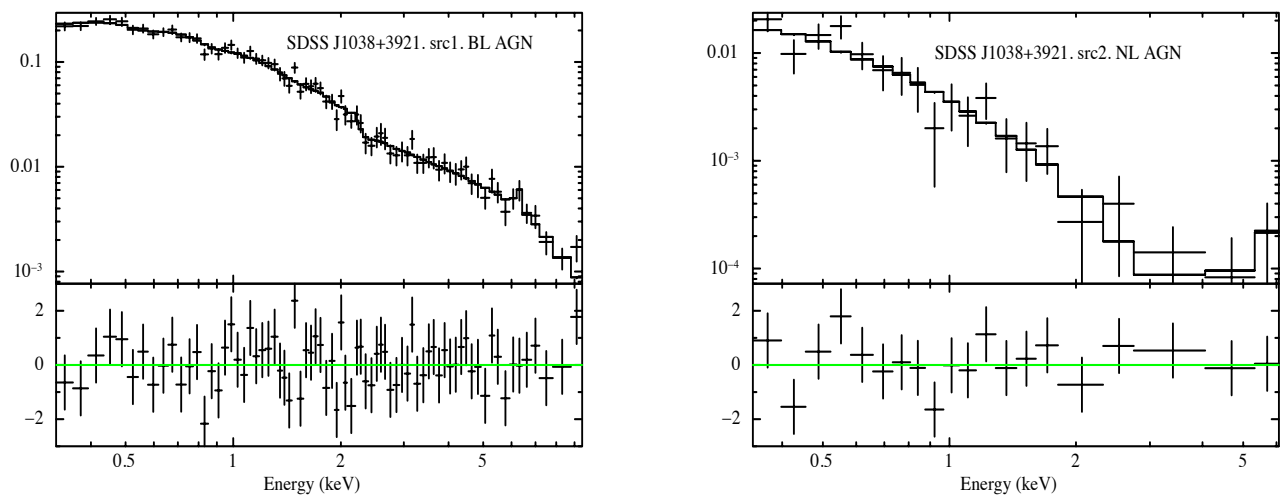

Fig. 5 SDSS J1038+3921. XMM-pn data of the sources detected in the system. Bottom panels show residuals of the best fit models and our data set in units of $\sigma$ (see details in Sect. 4). No strong residuals are present across the whole energy band.

tems with a total exposure of $180 \mathrm{ks}$ ). The observation of the second system has been already performed and a preliminary analysis confirmed the presence of both nuclei. Detailed analysis are in progress. The systems investigated so far and described in this paper have shown the effectiveness of our approach in order to study and characterize multiple AGN systems. These results clearly show the importance of snapshot ( $20-40 \mathrm{ks}$ ) observations with XMM and Chandra. The X-ray study potentially allows the detection and characterization of systems that are often elusive in the optical band. Thanks to its superior effective area up to $10 \mathrm{keV}$, $\mathrm{XMM}$ is particularly suited to detect the nuclei of systems at large separations, even in the case of strong absorption while the low background and sharp PSF of chandra will allow the study of systems in a close separation $(\lesssim 10 \operatorname{arcsec})$.
Haehnelt, M.\& Kauffmann, G. 2002, MNRAS, 336, L61

Hickson, P. 1997, ARAA, 35, 357

Jiménez-Bailón, 2007, A\&A, 469, 881

Nagar N.M.,et al., 2005, A\&A, 435, 521

Komossa, S. \& Zensus, J. A. 2016, IAUS, 312, 13

Komossa, S., et al. 2003, ApJ, 582 L15

Koss et al. 2011, ApJ 735 L42

Lanzuisi, G., et al. 2015, A\&A, 578, 120

Liu, X.; et al., 2011, ApJ, 737, 101

Piconcelli, E., et al. 2010, ApJ, 722, L147

Ribeiro, A. L: B., De Carvalho, R. R., et al., 1996, ApJ, 463, L5.

Rodriguez, C., et al., 2006, ApJ, 646, 49

Satyapal, S., et al., 2014, MNRAS, 441, 1297

Smith, K.L., et al. 2012, ApJ, 752, 63

Terashima \& Y., Wilson, A.S., 2003, ApJ, 583, 145

Turner M. J. L. et al., 2001 A\&A, 365, L110

Volonteri, M., et al. 2003, ApJ 582, 559

Acknowledgements. All coauthors, members of the MAGNA team (www.issibern.ch/teams/agnactivity/Home.html) acknowledge support of ISSI-Bern, Switzerland.

\section{References}

Baldwin et al. 1981, PASP, 93, 5

Becker, R. H., White, R. L., Helfand, D. J. 1994, ASPC, 61, 165

Begelman et al.1980, Nature 287, 307

Bianchi, S. et al. 2008, MNRAS, 386, 105,

Bogdanovic, T., 2015, ASSP, 40, 103

Bruzual G., Charlot S., 2003, MNRAS, 344, 1000

Cappi, M., et al. 2006, A\&A, 446, 459

Condon, J. J., wt al., 1998, AJ, 115, 1693

Comerford, J., et al. 2012, ApJ, 753, 42

De Rosa, A., et al. 2015, MNRAS, 453, 214

Escala et al. 2004, ApJ, 607, 765

Feltre A, et al., 2012. MNRAS 426

Fritz J, et al., 2006. MNRAS 366:767786

Fu, H., et al. 2011, ApJ, 733, 103

Fu, H., et al. 2012, ApJ, 745, 67

Gandhi, P. et al., 2009, A\&A 502, 457

González-Martín, O, et al. 2009, A\&A, 506, 1107

Guainazzi, M., et al 2005, A\&A 429, L9

Guainazzi, M. \& Bianchi, S., 2007, MNRAS, 374, 1290 
Table 1 The sources of the multiple systems analysed in this paper: SDSS J0959+1259 and SDSS J1038+3921.

\begin{tabular}{cccccccc}
\hline & $\begin{array}{c}\text { source } \\
\text { SDSS }\end{array}$ & $\begin{array}{c}\text { angular sep. } \\
(1)\end{array}$ & $\begin{array}{c}\text { proj. distance } \\
(2)\end{array}$ & $\begin{array}{c}\text { Z } \\
(3)\end{array}$ & $\begin{array}{c}\text { Lx } \\
(4)\end{array}$ & $\begin{array}{c}\text { L(OIII) } \\
(5)\end{array}$ & $\begin{array}{c}\text { type } \\
(6)\end{array}$ \\
\hline I-src1 & J095906.68+130135.4 & 327 & 227 & 0.037 & 5 & 0.07 & NL AGN \\
I-src2 & J095903.28+130220.9 & 377 & 262 & 0.036 & 0.12 & 0.003 & LINER \\
I-src3 & J095908.95+130352.4 & 318 & 221 & 0.0339 & 0.1 & 0.0008 & LINER \\
I-src4 & J095912.19+130410.5 & 280 & 195 & 0.0337 & $<0.3$ & 0.002 & SFG \\
I-src5 & J095914.76+125916.3 & 259 & 180 & 0.034 & 2.8 & 0.4 & NL AGN \\
I-src6 & J095859.91+130308.4 & 430 & 299 & 0.035 & $<0.2$ & 0.008 & SFG \\
I-src7 & J095900.42+130241.6 & 418 & 290 & 0.035 & $<0.1$ & 0.02 & SFG \\
I-src8 & J095955.84+130237.7 & 389 & 270 & 0.035 & 15 & 0.03 & BL AGN \\
\hline II-src1 & J103853.29+392151.2 & 40.4 & 42.9 & 0.0548 & 3.4 & 0.04 & BL AGN \\
II-src2 & J103855.95+392157.6 & - & - & - & $4^{\star}$ & 0.1 & NL AGN
\end{tabular}

(1)Angular separation between pairs in arcsec. In the case of SDSS J0959+1259 it is measured with respect to the center of the system $\left(149.87^{\circ} ; 13.03^{\circ}\right)$ (2)Projected distance in kpc. (3)Redshift. (4) $2-10 \mathrm{keV}$ unabsorbed luminosity in $10^{42} \mathrm{erg} \mathrm{s}^{-1}$. $^{\star}$ Assuming a Compton thick spectrum with $\mathrm{N}_{H}=10^{24}$ $\mathrm{cm}^{-2}$. (5) De-reddened [OIII] luminosity in $10^{42} \mathrm{erg} \mathrm{s}^{-1}$. (6) Object Type. 